Recibido: 12/octubre/2017 - Aprobado: 1/diciembre/2017

\title{
LA GESTIÓN ESTRATÉGICA DE LA INNOVACIÓN EN LAS ENTIDADES TURÍSTICAS DEL DESTINO LA HABANA.
}

MS.c. Yureidys García Leonard. ${ }^{1}$ \& Lic. Liety Lissett Alvarez Vázquez. ${ }^{2}$

\section{RESUMEN}

La innovación es un factor clave para lograr la competitividad. Además, incide en el posicionamiento y desarrollo de los destinos turísticos. En Cuba existen diferentes barreras que limitan el desarrollo del destino, impidiendo que el mismo sea innovador. Partiendo de la definición del perfil de viaje de los turistas, se puede enfocar una estrategia coherente en materia de innovación hacia el interior de las entidades, ya que, en empresas de servicios es vital el desarrollo de interface con los clientes, pues en gran medida, ellos aportan a la construcción y el desarrollo de las nuevas experiencias y los cambios. Inducir las actividades de I+D+i en la gestión de las entidades turísticas en el destino La Habana es una necesidad ante la muestras queda el agotamiento del modelo de desarrollo turístico sustentado en el "todo incluido".

PALABRAS CLAVE: Innovación, Destinos Turísticos Inteligentes, La Habana, $\mathrm{I}+\mathrm{D}+\mathrm{i}$.

\begin{abstract}
Innovation is a key factor to achieve competitiveness. In addition, it affects the positioning and development of tourist destinations. In Cuba, there are different barriers that limit the development of the destination, preventing it from being innovative. Starting from the definition of the tourist profile of the tourists, a coherent strategy in the field of innovation can be focused towards the interior of the entities, since, in service companies, the development of interface with the clients is vital, since to a great extent, they contribute to the construction and development of new experiences and changes. Induce $\mathrm{R}+\mathrm{D}+\mathrm{i}$ activities in the management of tourist entities in the destination Havana is a must before the samples is the exhaustion of the tourism development model supported by the "all inclusive"
\end{abstract}

\footnotetext{
${ }^{1}$ Universidad de la Habana, Facultad de Turismo, Cuba, ygarcia@ftur.uh.cu

${ }^{2}$ Ministerio de Turismo, La Habana, Cuba, lalvarez@ftur.uh.cu 
KEYWORDS: Innovation, Smart Tourist Destinations, Havana, I+D+i.

\section{INTRODUCCIÓN.}

Las economías insulares, como es el caso de la cubana, se perfilan como unos de los destinos más atractivos para desarrollar el Turismo bajo la modalidad de "sol y playa" que aunque se cuenta con potencialidades para el desarrollo de la misma, su modelo de gestión el "todo incluido", no es uno de los que permite la mayor captación de divisas, siendo este último uno de los objetivos pautados en la política económica del país, de ahí que lograr su desarrollo sobre la base de exportaciones con alto valor agregado precisa crear nuevos productos turísticos y estos no puede sustentarse, si no es a través de la inducción de actividades de innovación en las empresas enclavadas en el destino turístico que son actores esenciales en lograr la construcción de experiencias satisfactorias para los turistas.

Destino como La Habana, capital de Cuba, solventar un modelo de desarrollo turístico pasa por la apuesta al impulso de otras modalidades turísticas y no propiamente "Sol y Playa", modelo imperante en el destino país, sus principales atractivos no son justamente la belleza de sus playas, por lo que las entidades enclavadas en el mismo necesitan lograr una oferta turística asentada en el empleo del patrimonio arquitectónico, en el diseño de producto que aporten valor a los visitantes.

Siendo así las entidades encargada de dar la acogida y la hospitalidad en el destino constituyen un elemento esencial en la creación de productos turísticos innovadores, esta realidad en la práctica impone presión al desarrollo del destino, la gestión empresarial de estas, aun se basa en modelos tradicionales que no incorporan a la innovación dentro de sus procesos, por lo que el objetivo de la investigación es analizar el comportamiento de las entidades y definir los factores que determinan a la innovación. No si antes exponer los conceptos esenciales que defienden la propuesta final del artículo.

\section{La innovación. Particularidades en el sector de los servicios.}

Innovar es crear, cambiar o incrementar y que esta se materializa en nuevos productos, servicios y procesos, (Libro verde de la innovación, 1995), (Frascasti, 1992), (Oslo, 2005), (Manual de Bogotá, 2001) y (Arzola y D’ Armas, 2012), es un proceso de aprendizaje que utiliza al conocimiento como input y output, lo que contribuye a la formación de conceptos y teorías, (Vaquero, 1999), (Sánchez, 2009), (Benavides, C y Quintana, C, 2005), (Drucker, 1985), (López O, Blanco, M \& Guerra, S, 2009), (Nuñez, 
2013), (Elser, 1992 ), (Escorsa, 2003), (COTEC, 2010), (Bueno, 2012) y (Ahmed,K; Sheperd, C, 2012) resumiendo constituye una fuente de avance y desarrollo.

Autores diversos la defienden como el nuevo paradigma de la competitividad Vaquero (1999), Sánchez, (2008). Porter (2011) las economías de escalas y la productividad del trabajo son un viejo paradigma para lograr la competitividad de las naciones, que han sido superado por el único que existe hoy para crear ventajas competitivas, la Innovación, a través de la creación, diferentes caminos y nuevas combinaciones.

Drucker (1998) la concibe no solo como un producto de la investigación, éxitos y fracaso son fuente de inspiración en las empresas y punto de partida para generar un proceso que es una búsqueda intencionada de oportunidades ${ }^{3}$, ideas como estas fundamentan la necesidad de gestionar a la innovación y a la tecnología desde la empresa. Orientada al estudio de los procesos de innovación en el ámbito empresarial y su integración dentro de la estrategia corporativa como elemento clave para el éxito organizacional (Manjarres \& Vegas, 2012; Escorsa \& Valls, 1998, Ahmed \& Sheperd, 2012 y Shupe \& Behling 2006).

Más contemporáneo la asociación española de normalización y certificación (AENOR), la define como "parte del Sistema de gestión que incluye estructura organizativa, planificación de actividades, responsabilidades, prácticas, procedimientos, procesos y recursos para desarrollar, implantar, llevar a efecto, revisar y mantener al día la política de I+D+i”. (AENOR, 2016, pp 6.)

En resumen, la gestión de la innovación es parte intrínseca a los modelos de dirección empresarial por ello, dentro de la estrategia empresarial, la estrategia tecnológica juega un papel esencial. Esta última busca que la tecnología se convierta en el vehículo ideal en la creación de ventajas competitivas, desagregada en toda la cadena del valor de la entidad.

Hablar de innovación no requiere una distinción entre la actividad productiva y los servicios, genéricamente es el mismo proceso para ambas actividades, sin embargo, el sector terciario de la economía tiene singularidades que distingue el proceso a lo que normalmente se observa en la industria manufacturera.

Tres son los enfoques predominante en la literatura encargados de guiar el estudio de la innovación en los servicios: el primero basado en los aportes de Barras, se defiende la tesis de que no se constan diferencias sustanciales entre la manera de ver el proceso de innovación entre ambos tipos de actividad económica; un segundo enfoque de demarcación o divergente, considera las especificidades de los servicios, y hacen

\footnotetext{
${ }^{3}$ Para Drucker son oportunidades de innovación: 1. Acontecimientos inesperados, 2. Incongruencias. 3. Necesidades del proceso y 4. Cambios en el mercado. 
énfasis en sus propias características que limitan la capacidad de definir y medir la calidad de los productos y la productividad de las empresas de la misma manera que en otras industrias, por lo que es necesario desarrollar marcos específicos para entender al sector; una perspectiva conciliadora a las anteriores es el enfoque de síntesis, de integración o convergente, reconoce las diferencias entre la innovación en los servicios y en la industria manufacturera, pero manteniendo un punto de vista integrador que permite incorporar las características de ambos sectores uno de sus exponentes más recurrente es Gallouj (Crespi \& Olivari; 2016).

Aunque coexisten criterios divergentes en cuanto al estudio de la innovación en los servicios, se impone el criterio de síntesis como uno de los más prometedores para explicarla, teniendo en cuenta siempre la tendencia a ser un proceso más informal, poco sistémico y difícil de diferencial, (Elche, 2004; COTEC, 2014).

Estudios empíricos realizados en América Latina por Crespi y Olivari, arrojan que las empresas de servicios se ven enfrentadas a una serie de obstáculos que disminuyen su apego por la innovación, especialmente los vinculados a costos, conocimiento y mercado, siendo el primero el que parece tener una incidencia mayor, se observa que los factores vinculados al conocimiento tienden a ser más importantes para las empresas de servicios que para las de manufactura, mientras que los factores de mercado tienen mayor relevancia para las empresas de servicios que para las de manufactura. (Crespi \& Olivari, 2016).

Entender a la innovación en el servicio implica concebirla como un proceso de cambio continuo, una combinación de creatividad y aprendizaje, en constante interface con clientes y proveedores, cuyo fin es la satisfacción del cliente. (Sundbo \& Gallouj, 2015), con una tendencia a un proceso informal, flexible y gestionado por grupos de proyecto. (Elche, 2004).

Modelos diseñados para aplicar la innovación de empresas de servicios, captan la idea del anterior párrafo se destacan: Sundbod (1998), Bilderberg (1999), AENOR (2006), Arzolas (2007), Igaratua (2009), COTEC (2010), Vargas (2013), INOSEV (2014) y Sundbo \& Gallouj (2015). Estos nos permiten sistematizar aspectos a tener en cuenta cuando se estudie la innovación en los servicios tales como:

1. El desarrollo de la inteligencia competitiva y de mercado como etapas fundamentales del proceso.

2. La importancia del enfoque prospectivo y estratégico y el nexo del desarrollo tecnológico con la estrategia empresarial.

3. El desarrollo organizacional y la mejora continua de los procesos internos como fuente fundamental de innovación.

4. El capital humano, su motivación y desarrollo dentro de la entidad. 
Específicamente en el turismo Halager apunta que las políticas en materia del cambio climático serán una fuerza inductora importante para el desarrollo de las innovaciones en el mismo (Halager, 2010).

En el 2015, Sundbo, realizó un estudio empírico sobre la innovación en el turismo, basó su análisis en tres niveles: el empresarial, el de red y los sistemas.

Como resultado del estudio destaca que la actitud innovadora de las empresas de este sector está directamente ligada al tamaño, que son más propensas a innovar la que desarrolla un enfoque estratégico y se centran en el desarrollo y calificación del capital humano, las que trabajan en redes locales y globales.

Resume que la innovación en el turismo no puede ser explicada por factores tecnológicos o por la determinación de los proveedores, debe de ser explicada por factores sociales y una política clara.

Sin embargo, la innovación en el turismo se enfrenta a una serie de barreras que le impiden un mayor desarrollo:

- Atomización empresarial, que dificulta la financiación, la disponibilidad de personal cualificado y la asunción de los riesgos económicos asociados a la innovación; además de suponer mayores resistencias al cambio.

- Déficit de cultura de la innovación.

- Falta de fuentes de financiación. Involucración empresarial.

- Dificultades en la transmisión y adopción del conocimiento.

- Carencia de un enfoque sistemático de la innovación del que sí disponen otros sectores industriales.

- Dificultad de medir la innovación por las características de la actividad turística.

- Desigual implantación de la innovación por subsectores turísticos ${ }^{4}$.

\section{Estudio de las particularidades de la innovación en entidades del destino La Habana.}

Para llevar a cabo el estudio se aplicó un cuestionario a las 65 entidades pertenecientes a la 7 OSDE del Ministerio del Turismo, exceptuando a las entidades de Ocio y Recreación. El análisis de fiabilidad del instrumento aplicado arrojó un Alfa de Crombach, de 0.966, demostrando la alta fiabilidad del instrumento de medición y la consistencia interna de la escala de medición que se utilizó.

\footnotetext{
${ }^{4}$ Para ver un estudio más exhaustivo del tema consultar el Manual Operativo para La Configuración de Destinos Turísticos Inteligentes 


\section{Factores que inciden en la gestión de la innovación en las entidades turísticas como un proceso estratégico.}

La baja disposición innovadora de las entidades turísticas encuestadas, no es producto del desconocimiento o la poca prioridad que se le confiere dentro del modelo de dirección implementado, asocian la poca expectativa a innovar problemas de financiamiento, no se tienen en cuenta un presupuesto asociado a la actividad de investigación, lo que limitan el desarrollo de nuevos productos, al no contarse con partidas de gastos necesarias en la etapa de investigación y desarrollo.

Corroboran lo anterior que el $100 \%$ de las encuestadas reconocen que en las normas estipuladas constan los fundamentos para desarrollar una política de innovación a escala empresarial.

Siendo así, los problemas no están centrado en la institucionalidad de la actividad y si hacia los mecanismos sectoriales y microeconómicos en cuanto a la respuesta de la pregunta de cómo implementarla.

Las principales concepciones insisten en el desarrollo de redes y colaboraciones, como un estadio inicial en la concreción de un sistema de innovación como el marco propicio en logro de un entorno innovador, las empresas estudiadas no muestran actitudes colaborativas y si un comportamiento competitivo entre ellas, teniendo un tamaño pequeño y una cuota de mercado poco significativa.

Hacia el interior de su desempeño, no utilizan proceso de vigilancia, jerarquizan la creación de nuevo productos como forma de innovación y desconocen que nuevas formas de distribución, el diseño de nuevos procesos, ni nuevos modelos de gestión son fuente de esta.

El comportamiento de la muestra es homogéneo por tipo de entidad no existe diferencia entre las respuestas de los hoteles, los restaurantes y las agencias de viajes, solamente en el aspecto en cuanto a la realización de nuevos productos, las agencias de viajes y los restaurantes tienen una mejor conducta que los hoteles, sin embargo son estos últimos lo que mejor responden en cuanto al empleo de nuevos proceso y nuevas formas de distribución, de lo que se puede inferir que el tamaño y el tipo de actividad no son factores que influyen en un comportamiento en cuanto a las expectativas empresariales para innovar.

Sin embargo utilizando como punto de análisis el paradigma estructura-conductaresultado, la estructura del sector del turismo en el destino estudiado, condiciona su conducta en la medida que funciona como un mercado de competencia monopolista, 
donde el tamaño de las empresas es pequeño hay una cantidad representativa de ellas y bajas barreras de entrada, su comportamiento se centran en temas competitivos de diferenciación del producto, mostrando poca colaboración elemento esencial en la innovación.

El Modelo de COTEC (1999) plantea el uso de herramientas atendiendo a los cincos objetivos para gestionar a la innovación en las empresas, en la tabla que se presenta a continuación se realiza un diagnóstico de las entidades analizadas a raíz de los resultados de las encuestas.

Tabla 1. Deficiencias que presentan las entidades del turismo.

\begin{tabular}{|c|c|c|}
\hline Vigilancia & $\begin{array}{l}\text { DAFO } \\
\text { Investigación de Mercado } \\
\text { Prospectiva } \\
\text { benchmarking }\end{array}$ & $\begin{array}{l}\text { Deficiente sistema de inteligencia } \\
\text { empresarial. }\end{array}$ \\
\hline Focalizar & $\begin{array}{l}\text { La auditoría de capacidades } \\
\text { Modelos de Carteras. } \\
\text { Modelos de proyectos }\end{array}$ & $\begin{array}{l}\text { No se encuentra desarrollada en las } \\
\text { entidades }\end{array}$ \\
\hline Capacitación & $\begin{array}{l}\text { Gestión del conocimiento } \\
\text { Gestión de la tecnología } \\
\text { Gestión de los recursos } \\
\text { Humanos }\end{array}$ & $\begin{array}{l}\text { Enfocada hacia lo profesional, a la } \\
\text { asimilación de las TIC }\end{array}$ \\
\hline Implantación & $\begin{array}{l}\text { Gestión de proyectos } \\
\text { Gestión del cambio } \\
\text { Gestión de interfaces } \\
\text { Implantación de la calidad } \\
\text { total } \\
\text { Colaboración externa }\end{array}$ & $\begin{array}{l}\text { En este objetivos las entidades no } \\
\text { cuentan con las capacidades } \\
\text { creadas para emprenderlo }\end{array}$ \\
\hline $\begin{array}{l}\text { Aprendizaje y } \\
\text { Mejora continua }\end{array}$ & $\begin{array}{l}\text { La evaluación } \\
\text { Y el enfoque de mejora } \\
\text { continua }\end{array}$ & $\begin{array}{l}\text { La calidad no se utiliza como } \\
\text { instrumento estratégico dentro de } \\
\text { la empresa. }\end{array}$ \\
\hline
\end{tabular}

Fuente. Elaboración propia.

Como se pudo apreciar en la práctica de las entidades existen las norman y los documentos que rigen lo que debe de propiciar un clima innovador, en la praxis sucede todo lo contrario no hay una comprensión real del fenómeno y se confronta que existe una baja cultura innovadora 
El estudio anterior demuestra que la baja actividad innovadora de las empresas turística, amén de los factores objetivos como es la financiación de los proyectos de investigación, la inexistencia de una clara política sectorial de innovación, la debilidad del sistema nacional de innovación que no se traduce en sistema sectoriales, pasa por problemas no solo estructurales, sino aspectos más subjetivos en la gestión, como por ejemplo: el comportamiento de los arribos que muestran un crecimiento en el destino, induce una respuestas pasiva en las entidades, que no ven la necesidad de renovarse, indicadores los ingresos muestran una tendencia creciente, no obstante, a esta situación se impone buscar nuevas formas, las tendencias avanzan y se continua apostando por una forma de hacer turismo de manera tradicional y una gestión excesivamente reactiva hacia el interior de las organizaciones.

Lo dicho anteriormente impone reto a la gestión del destino tales como:

- Trabajar sobre el diseño de los sistemas de inteligencia empresarial.

a) Proponer a la vigilancia tecnológica como una función dentro de las entidades y paso inicial para consolidar un sistema de inteligencia competitiva.

b) Diseñar un sistema de indicadores que capten la información necesaria para consolidar bases de datos.

c) Perfeccionar los sistemas de información de las entidades

- Diseñar una clara política de innovación desde el Ministerio y su derivación hacia las entidades.

- Concebir a la estrategia tecnológica dentro de la corporativa

- Capacitar a las entidades en tema de:
a. Gestión de proyectos.
b. Ingeniería de datos.
c. Herramientas de gestión empresarial.

En la siguiente figura se muestra los procesos esenciales a desarrollar en las entidades para inducir una cultura innovadora a escala microeconómica. 
Figura 1. Procesos esenciales dentro de la entidad para lograr la gestión de la innovación.

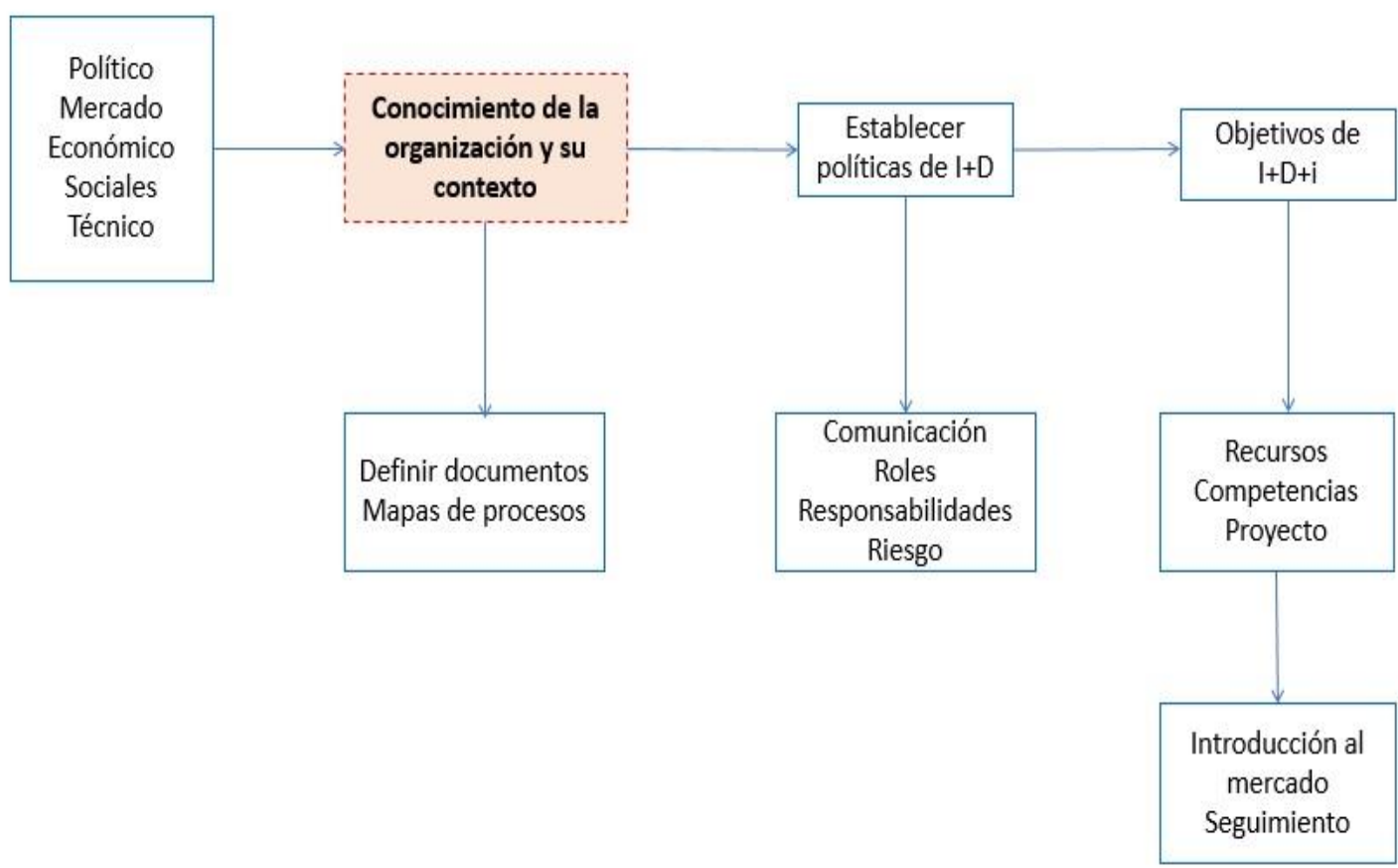

Fuente: Elaboración propia.

\section{CONCLUSIONES}

- Las entidades enclavadas en el destino no conciben a la innovación dentro del sistema de gestión empresarial, aunque existen normas y decretos para constituirla como un proceso esencial en la dirección.

- Los factores que inciden en la baja conducta innovadora de las entidades pasa por aspectos objetivos, preocupa sin embargo que el problema es mucho más complejo pues no se interioriza la importancia del tema y las principales decisiones de desarrollo se siguen tomando sobre la base del comportamiento de indicadores con un sesgo para medir a la actividad turística

- Finalmente la gestión y desarrollo de los recurso humanos desde las entidades no constituye un factor decisivo para inducir procesos de innovación en la organización al predominar la profesionalización en materia de capacitación quedando relegada las habilidades necesarias para la innovación. 


\section{REFERENCIAS BIBLIOGRÁFICAS}

AENOR. (2006). Norma UNE 166002. Obtenido de http://www.scielo.org.co/scielo.php?script=sci_serial\&pid=0121$4772 \& \operatorname{lng}=$ en $\&$ nrm $=$ iso

AENOR. (2016). Gestión de la I+D+i: El proceso de certificación de un Sistema de Gestión de la $I+D+i$. Galicia.

Ahmed, P., \& Sheperd, C. (2012). Administración de la innovación. México DF.

Arocena, R., \& Sutz, J. (2013). Innovación y democratización del conocimiento como contribución al desarrollo inclusivo. Sistema de innovación para un desarrollo inclusivo. La experiencia latinoamericana. Ciudad México.

Arzola, M. (2007). Innovación, competitividad y desarrollo. Valencia, Venezuela.

Arzola, M., \& D’Armas, M. (2012). Análisis comparativo de los modelos de gestión para la innovación en las organizaciones empresariales.

Benavides, C. A., \& Quintana, C. (2006). Inteligencia competitiva, prospectiva e innovación. La norma UNE-166006 EX sobre el sistema de vigilancia tecnológica. Boletín Económico de ICE $N^{\circ} 2896$.

Bilderbeek, R. (1999). Conceptualising Service Innovation and Service Innovation Patterns.

Bueno, E. (2010). El gobierno del conocimiento organizativo: un análisis interdisciplinar y una realidad multidisciplinar de naturaleza compleja.

Bueno, E. (2012). El capital Intelectual de las Organizaciones. Madrid: AECA.

CEPAL. (2014). Nuevas instituciones para la innovación. Prácticas y experienias en América Latina.

Comisión Europea. (1995). Libro Verde de la Innovación.

COTEC. (2010). La innovación en sentido amplio: un modelo empresarial.

COTEC. (2014). Tecnología e innovación en España. Madrid. Obtenido de http://www.cotec.es

Crespi , \& Olivari. (2016). La política de innovación en América Latina y el Carbe. Nuevos Caminos. Obtenido de https://publications.iadb.org/.../La-politica-deinnovacion-en-America-Latina-y-el-Carib...

Drucker, P. (1985). The Discipline of Innovation. Harvard Business Review.

Drucker, P. F. (1998). The discipline of innovation. Harvard Business Review.

Elche, M. D. (2004). La innovación en los servicios: análisis de la relación de tipo de servicios-patrón de innovación y su incidencia en el resultado. Departamento de Economía y Empresa. Cuenca: Ediciones de la Universidad de Castilla-La Mancha. 
Escorsa, P., \& Valls, J. (1998). Tecnología e innovación en la empresa.

Escorsa, P., \& Valls, J. (2003). Tecnología e innovación en la empresa (Segunda Edición ed.). Barcelona: Ediciones UPC.

Gadduf, J., \& García, A. (1999). Política económica y actividad empresarial. Universidad de Valencia: Tirant lo Blanch.

Hertog, P., \& Bilderbeek, R. (1999). Conceptualising Service Innovation and Service Innovation Patterns.

Hjalager, A.-M. (2010). Progress in Tourism Management. A review of innovation research in tourism. Tourism Management.

Igartua, J. I. (2009). Gestión de la innovación en la empresa vasca: Contribución de las herramientas de gestión de la innovación. Tesis doctoral, Universidad Politécnica de Valencia, Departamento de Organización de Empresas.

ISEA. (2014). Modelo INNOSERV, para el Diseño e Implantación de un Sistema de Gestión de la Innovación en el Sector de Servicios.

Kline, S. (1985). Innovation is not linear process. Research Management, 36-45.

López, O., Blanco, M., \& Guerra, S. (2009). Evolución de los modelos de la gestión de la innovación. Innovación de negocios., 5(2), 251-264.

Manjares, \& Vegas. (2012). La gestión de la innovación en la empresa: evolución de su campo de estudio. Dimensión empresarial., 10(1), 18-29. Obtenido de https://www.uac.edu.co/images/stories/publicaciones/revistas_cientificas/dimension -empresarial/volumen-10-no-1/articulo02.pdf

Manual de los Destinos Inteligentes. (2015). Sociedad Estatal para la Gestión y las Tecnologías Turísticas. Recuperado el 15 de 04 de 2016, de http://www.segistur/informe_anual.es

Medellín, E. A. (2010). Gestión Tecnológica en Empresas Innovadoras Mexicanas. Revista de Admistración e Innovación, vol. 7(3), 58-78.

Nuñez, J. (2012). Educación superior, innovación y desarrollo local: Experiencias en Cuba. En G. Dutrenit, \& J. Sutz, Sistema de innovación para un desarrollo inclusivo. La experiencia latinoamericana. (págs. 227-252). Distrito Federal, México.

OCDE. (2002). Manual de Frascasti. Recuperado el 2015, de http://www.oecd.org/centrodemexico/publicaciones/

OCDE. (2005). Manual de Oslo. Obtenido de http://www.oecd.org/centrodemexico/publicaciones/

Porter, M. (2011). Creating Toorrow's Advantages. 
RICYT. (2001). Manual de Bogotá. Normalización de indicadores de innovación tecnológica en América Latina y el Caribe. Obtenido de http://www.ricyt.org/component/docman/cat_view/16-manuales?Itemid=2

Sánchez, R. (2009). La nueva economía y el conocimiento: entre el mito y la realidad. La Habana: Editorial Félix Varela.

Schumpeter, J. (1934). The theory of Economic Development (Vol. vol. XVII). Harvard University Press.

Seggitur. (2015). Libro Blanco de los Destinos Turísticos Inteligentes. Madrid.

Sundbo, J. (1998). Innovation Theory: Three Paradigms. .

Sundbo, J., \& Gallouj, F. (2015). Innovation in Services. Synthesis report(2).

Vargas, L. E. (2013). Diseño de una propuesta metodológica para gestionar la innovación en empresas desarrolladoras de software integrantes de la organización Network Clúster TIC del Triángulo del Café. Trabajo de investigación para optar al título de Magister en Administración, Universidad Nacional de Colombia, Facultad de Administración, Manizales.

Vázquez, A. (1999). Desarrollo, redes e innovación. Lecciones sobre desarrollo endógeno. Madrid: Pirámide, SA.

Velasco, E., Zamanillo, I., \& Gurutse, M. (2006). Evolución de los modelos sobre el proceso de innovación: desde el modelo lineal hasta los sistemas de innovación. (Vol. 2). Mallorca.

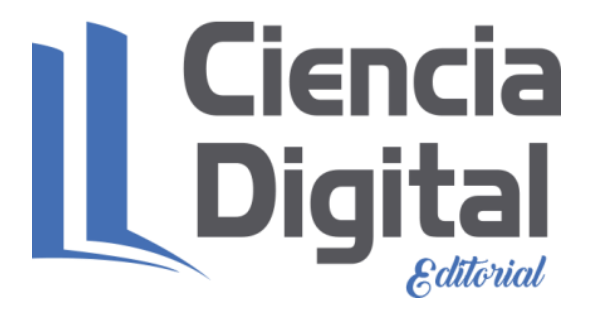




\section{PARA CITAR EL ARTÍCULO INDEXADO.}

García Y. \& Alvarez L. La gestión estratégica de la innovación en las entidades turísticas del destino la Habana. Revista electrónica Visionario Digital 1(1), 5-18. Recuperado desde: http://www.visionariodigital.org/index.php/VISIONARIODIGITAL/article/view/40/47

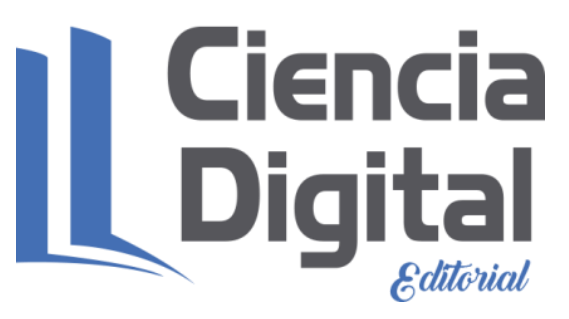

El artículo que se publica es de exclusiva responsabilidad de los autores y no necesariamente reflejan el pensamiento de la Revista Ciencia Digital.

El articulo queda en propiedad de la revista y, por tanto, su publicación parcial y/o total en otro medio tiene que ser autorizado por el director de la Revista Ciencia Digital.
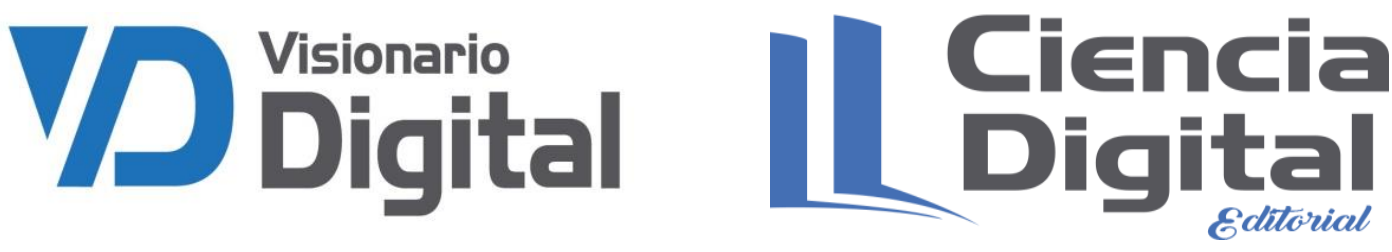